\title{
Late accretion of volatiles to a dry proto-earth: really?
}

\author{
DR. JABRANE LABIDI ${ }^{1}$, JAMES DOTTIN III ${ }^{2}$, AIERKEN \\ YIERPAN $^{3}$, ANAT SHAHAR ${ }^{4}$, PIERRE CARTIGNY ${ }^{5}$, \\ JAMES FARQUHAR $^{2}$ AND EDWARD YOUNG ${ }^{6}$
}

\author{
${ }^{1}$ Institut de Physique du Globe de Paris \\ ${ }^{2}$ University of Maryland \\ 3 tuebingen university \\ ${ }^{4}$ Carnegie institution for science \\ ${ }^{5}$ IPGP \\ ${ }^{6}$ University of California, Los Angeles \\ Presenting Author: labidi@ipgp.fr
}

Terrestrial volatiles may have been acquired by a late veneer of carbonaceous chondrite-like material impacting the protoEarth after core formation was completed. However, volatile exchange between the surface and the deep Earth has likely clouded some of the late-veneer signatures. In this talk, we will present recent advances in our understanding of the isotope geochemistry of key volatile elements. A particular focus on sulfur, selenium, and nitrogen. allows us to assess whether the late veneer delivered substantial amounts of volatiles to Earth.

Mantle plume sources show variable sulfur isotope signatures. ${ }^{34} \mathrm{~S} /{ }^{32} \mathrm{~S}$ ratios appear correlated with proxies of recycling. The trends show that basalts with no clear evidence of recycled materials have a sub-chondritic ${ }^{34} \mathrm{~S} /{ }^{32} \mathrm{~S}$ ratio. Sulfur partitioning experiments suggest metal/silicate equilibria may be at play: the sub-chondritic $\mathrm{S}$ isotope composition observed in the modern mantle is likely a relic of planetary differentiation. This observation does not require a volatile-rich late veneer.

Selenium is often compared to sulfur, since S and Se likely behave similarly in high-temperature processes. High-precision ${ }^{82} \mathrm{Se} /{ }^{76} \mathrm{Se}$ data show basalts are more depleted in ${ }^{82} \mathrm{Se}$ than most carbonaceous chondrites. This is evidence against the delivery of volatiles by late accretion of carbonaceous chondrites.

Nitrogen is an atmophile, largely partitioned into the Earth's atmosphere. The Earth's mantle has a peculiar ${ }^{15} \mathrm{~N} /{ }^{14} \mathrm{~N}$ ratio from a meteoritical perspective, with values halfway between enstatite and carbonaceous chondrites. Estimates of modern $\mathrm{N}$ fluxes appear consistent with mantle $\mathrm{N}$ being attributable to subduction. If so, any primordial ${ }^{15} \mathrm{~N} /{ }^{14} \mathrm{~N}$ would have been erased over time. Recent measurements of the doubly-substituted isotopologue ${ }^{15} \mathrm{~N}^{15} \mathrm{~N}$ in volcanic gases have shown, however, that the nitrogen fluxes to the mantle may have been overestimated. The ${ }^{15} \mathrm{~N} /{ }^{14} \mathrm{~N}$ ratio of the Earth's mantle may in fact be vestiges of planetary formation rather than inheritance from the surface. The planetary $\mathrm{N}$ isotope composition is also not consistent with a carbonaceous late veneer.

Overall, new isotopic observations appear to challenge the notion that volatiles were delivered by a volatile-rich late veneer to a dry proto-Earth. 